Pacific Journal of Mathematic 


\title{
THE TRANSFER OF INVARIANT PAIRINGS TO LATTICES
}

\author{
T. J. Enright and R. Parthasarathy
}

\begin{abstract}
In the article "On the fundamental series of a real semisimple Lie algeba" two covariant functors, the completion functor and the lattice functor, are introduced. In this article, we study the behavior of invariant pairings and invariant Hermitian pairings under the action of these functors.
\end{abstract}

Let $\mathfrak{m}$ be a finite dimensional reductive Lie algebra over $\boldsymbol{C}$, the field of complex numbers, and let $U(\mathfrak{m})$ denote its universal enveloping algebra. Let $\mathfrak{h}$ be a CSA of $\mathfrak{m}$ with roots $\Delta$, a positive system $Q$ of $\Delta$ and Weyl group $\mathscr{W}$. For each $\alpha \in Q$, let $\mathfrak{m}_{\alpha}$ denote the $\alpha$ root space in $\mathfrak{m}$ and choose vectors $\bar{H}_{\alpha}, X_{\alpha}, X_{-\alpha}$ such that

$$
X_{\alpha} \in \mathfrak{m}_{\alpha}, \quad X_{-\alpha} \in \mathfrak{m}_{-\alpha}, \quad \bar{H}_{\alpha}=\left[X_{\alpha}, X_{-\alpha}\right], \quad \alpha\left(\bar{H}_{\alpha}\right)=2 .
$$

Then $\bar{H}_{\alpha}, X_{\alpha}, X_{-\alpha}$ is called a standard triple and spans a subalgebra $\mathfrak{a}^{(\alpha)}$ of $\mathfrak{m}$ isomorphic to $\operatorname{sl}(2)$. Choose and fix once and for all an involutive anti-automorphism $\sigma$ of $\mathfrak{m}$ such that $\sigma$ restricted to $\mathfrak{h}$ equals the identity. Fix a real form $\mathfrak{m}_{0}$ of $\mathfrak{m}$ such that $\left[\mathfrak{m}_{0}, \mathfrak{m}_{0}\right]$ is a compact real form of $[\mathfrak{m}, \mathfrak{m}]$ and $\mathfrak{m}_{0} \cap \mathfrak{h}=\mathfrak{h}_{0}$ is a real form of $\mathfrak{h}$. Let $\mathfrak{m}_{R}$ be the real Lie algebra underlying $\mathfrak{m}$ and let $\bar{\sigma}$ be the $R$-linear antiautomorphism of $U\left(\mathfrak{m}_{R}\right)$ uniquely determined by the condition that $-\bar{\sigma}$ restricted to $\mathfrak{m}$ equals the conjugation of $\mathfrak{m}$ with respect to $\mathfrak{m}_{0}$. For m-modules $A$ and $B$, a bilinear (resp. Hermitian) pairing $\langle\cdot, \cdot\rangle$ of $A$ and $B$ is called invariant if $\langle x \cdot a, b\rangle=\left\langle a, x^{\sigma} \cdot b\right\rangle$ (resp. $\langle x \cdot a, b\rangle=$ $\left.\left\langle a, x^{\bar{\sigma}} \cdot b\right\rangle\right), a \in A, b \in B, x \in U(\mathfrak{m})$. Denote by $I_{\mathrm{m}}(A, B)\left(\right.$ resp. $\left.I H_{\mathrm{m}}(A, B)\right)$ the $C$-linear (resp. $R$-linear) space of invariant (resp. invariant Hermitian) pairings of $A$ and $B$. If $g$ is any finite dimensional Lie algebra over $C$ with $\mathfrak{m} \subset \mathfrak{g}$ and if $\sigma_{\mathfrak{g}}$ (resp. $\bar{\sigma}_{\mathfrak{g}}$ ) is an involutive anti-automorphism of $U(\mathfrak{g})$ (resp. $U\left(\mathfrak{g}_{k}\right)$ ) which restricts to $\sigma$ (resp. $\bar{\sigma}$ ) on $\mathfrak{m}$, then we define $g$-invariant pairings as above with $\sigma$ replaced by $\sigma_{\mathrm{g}}$ (resp. $\bar{\sigma}$ replaced by $\left.\bar{\sigma}_{\mathfrak{g}}\right)$ and $U(\mathfrak{m})$ replaced by $U(\mathfrak{g})$. For $\mathfrak{g}$-modules $A$ and $B$, we denote by $I_{8}(A, B)$ (resp. $I H_{8}(A, B)$ ) the spaces of $\mathfrak{g}$-invariant (resp. g-invariant Hermitian) pairings of $A$ and $B$.

For each $\alpha \in Q$, let $C_{\alpha}$ denote the completion functor determined by the standard triple $\bar{H}_{\alpha}, X_{\alpha}, X_{-\alpha}$. If $\mathfrak{m}=s l(2)$ then we write $H$, $X, Y$ in place of $\bar{H}_{\alpha}, X_{\alpha}, X_{-\alpha}$ and denote the functor $C_{\alpha}$ by $C$ (there is only one positive root in this case). Also in this case for $n \in N$ (integer $\geqq 0$ ) and $A$ an $m$-module we write $A[n]$ for the subspace of $H$-eigenvectors with eigenvalue $n$ and $A^{X}[n]$ for the subspace of $A[n]$ 
of vectors mapped to zero by $X$. In the general case, Bouaziz [1] and Deodhar [3] have shown that for any g-module $A$ in the category $\mathscr{F}_{8}(\mathfrak{m})$ (cf. Definition 4.1 [5]) there is a lattice above $A$. Let $A_{s}(s \in \mathscr{W})$ denote this lattice above $A$ and define the functor $\tau$ by the formula: $\tau(A)=A_{1} / \sum_{s \neq 1} A_{s} . \quad \tau(A)$ is a $U(\mathfrak{m})$-finite $\mathfrak{g}$-module.

We can now state the main results of this article. First assume $\mathfrak{m}=\operatorname{sl}(2)$. For $A, B \in \mathscr{J}(\mathfrak{m})$ and $\varphi \in I_{\mathfrak{m}}(A, B)$ (resp. $I H_{\mathfrak{m}}(A, B)$ ) there exists a unique pairing $C(\varphi) \in I_{\mathfrak{m}}\left(C(A), C(B)\right.$ ) (resp. $I H_{\mathrm{m}}(C(A), C(B))$ ) such that

(i) $C(\varphi)$ is zero on $(A \times C(B)) \cup(C(A) \times B)$

(ii) for all $n \in N, a \in C(A)^{X}[n], b \in C(B)^{X}[n]$

$$
C(\varphi)(a, b)=\frac{1}{n !(n+1) !} \varphi\left(Y^{n+1} a, Y^{n+1} b\right) .
$$

This result is given below as Proposition 3.1 and is a mild variation of a result in [5] which concerns only invariant forms. The main result of $\S 3$ is Theorem 3.4 which asserts that if $A, B \in \mathscr{J}_{\mathrm{g}}(\mathfrak{m})$ and $\varphi \in I_{8}(A, B)$ (resp. $I H_{8}(A, B)$ ) then $C(\varphi) \in I_{8}\left(C(A), C(B)\right.$ ) (resp. $I H_{8}(C(A)$, $C(B))$ ). For $\varphi \in I_{8}(A, A)$, this theorem is precisely Proposition 8.5 [5]. The proof given there is based on an elaborate computation. The proof given in this article is more conceptual and is based on properties of certain vector valued pairings.

In $\S 4$ we apply the results of $\S 3$ to the setting of general reductive Lie algebras $\mathfrak{m}$. Let $t_{0}$ be the unique element of $\mathscr{W}$ such that $t_{0} Q=-Q$ and let $t_{0}=s_{\alpha_{1}} \circ \cdots \circ s_{\alpha_{d}}$ be a reduced expression for $t_{0}\left(\alpha_{i} \in Q\right.$, simple roots). Let $A, B \in \mathscr{F}_{8}(\mathfrak{n t})$ and $\varphi \in I_{8}(A, B)$ (resp: $I H_{g}(A, B)$ ). Then (cf. Proposition 4.2), the pairing $C_{\alpha_{1}} \circ \cdots \circ C_{\alpha_{d}}(\varphi)$ on $A_{1} \times B_{1}$ is independent of the reduced expression for $t_{0}$ and is zero on $\left(\sum_{s \neq 1} A_{s} \times B_{1}\right) \cup\left(A_{1} \times \sum_{s \neq 1} B_{s}\right)$. This pairing induces a pairing of $\tau(A)$ and $\tau(B)$ which we denote by $\tau(\varphi)$. Clearly, from the results of $\S 3, \tau(\varphi) \in I_{8}\left(\tau(A), \tau(B)\right.$ ) (resp. $I H_{8}(\tau(A), \tau(B))$ ).

Define the Borel subalgebra $\mathfrak{b}=\mathfrak{h} \oplus \sum_{\alpha \in Q} \mathfrak{m}_{\alpha}$. For any $\mathfrak{b}$-module $M$, we denote the induced module from $\mathfrak{b}$ to $\mathfrak{m}$ by $U(M)$; i.e., $U(M)=$ $U(\mathfrak{m}) \otimes_{U(\xi)} M$. The main result of this last section concerns an important example where the map $\varphi \mapsto \tau(\varphi)$ is surjective and preserves nondegenerate pairings. This result (Proposition 4.5) asserts the following:

Let $M$ and $N$ be locally finite $\mathfrak{b}$-modules which are semisimple as $\mathfrak{h}$-modules with finite dimensional integral weight spaces. Assume that $U(M)$ and $U(N)$ admit nondegenerate invariant forms. Then the maps:

$$
\begin{aligned}
& \tau: I_{\mathrm{m}}(U(M), U(N)) \longrightarrow I_{\mathrm{m}}(\tau(U(M)), \tau(U(N))) \\
& \tau: I H_{\mathrm{m}}(U(M), U(N)) \longrightarrow I H_{\mathrm{m}}(\tau(U(M)), \tau(U(N)))
\end{aligned}
$$


are surjections. Moreover, both maps carry nondegenerate pairings to nondegenerate pairings.

This result is an important part of the theory of the functor $\tau$. In particular, it is used in "The representations of complex semisimple Lie groups" [6] to show that every irreducible representation of a complex Lie group is infinitesimally equivalent to the image under $\tau$ of an irreducible highest weight module.

2. Notation. We continue with the notation of $\S 1$. Let $\mathfrak{n}=$ $\sum_{\alpha \in Q} \mathfrak{m}_{\alpha}$ and $\mathfrak{n}^{-}=\sum_{\alpha \in Q} \mathfrak{m}_{-\alpha}$. Then $\mathfrak{b}=\mathfrak{h} \oplus \mathfrak{n}$ and $\mathfrak{m}=\mathfrak{n}^{-} \oplus \mathfrak{b}$. For any $\mu \in \mathfrak{h}^{*}$, let $\boldsymbol{C}_{\mu}$ denote the one dimensional $\mathfrak{b}$-module corresponding to $\mu$ and let $V_{\mathrm{m}, Q, \mu}=U(\mathfrak{m}) \bigotimes_{U(6)} C_{\mu}$ denote the Verma module with highest weight $\mu$. For any m-module $A$, let $A^{*}$ be the contragradient module. Let $Z(\mathfrak{m})$ denote the center of $U(\mathfrak{m})$ and $Z(\mathfrak{m})^{\wedge}$ the set of homomorphisms of $Z(\mathfrak{m})$ into $C$. It is well known that $Z(\mathfrak{m})^{\wedge}$ is parametrized by the Weyl group orbits in $\mathfrak{h}^{*}$. For $\chi \in Z(\mathfrak{m})^{\wedge}$ and an m-module $A$, let $A_{\text {z }}$ equal the submodule of $A$ where $z-\chi(z) \cdot 1$ is locally nilpotent for all $z \in Z(\mathfrak{m})$. Let $A^{\mathfrak{n}}$ denote the $\mathfrak{h}$-submodule of $A$ of vectors mapped to zero by $\mathfrak{n}$; and, for $\mu \in \mathfrak{h}^{*}$, let $A^{\mathrm{n}}[\mu]$ be the subspace of $A^{\mathfrak{n}}$ of vectors of weight $\mu$. Let $U^{\mathfrak{m}}(\mathfrak{m})$ denote the usual filtration of $U(\mathfrak{m}), \mathfrak{m} \in N$. For complex vector spaces $A, B$ and $F$, and $F_{0}$ a real form of $F$, we say that $\phi$ is a Hermitian map of $A \times B$ into $F$ if $\varphi$ is linear in the first variable and conjugate linear in the second variable. If $A=B$, we say $\varphi$ is a Hermitian form if $\varphi$ is a Hermitian map and $\varphi(a, b)=\overline{\varphi(b, a)}, a, b \in A$ and denoting conjugation of $F$ with respect to $F_{0}$.

3. Invariant and invariant Hermitian pairings. Let notation be as in $\S 1$ except that we shall write $a$ in place of $\mathfrak{m}$ and assume $a \cong \operatorname{sl}(2)$. $H, X, Y$ will be a standard triple for $a$ and $C$ will denote the completion functor with respect to $H, X, Y$ defined on the categories $\mathscr{F}_{\mathfrak{s}}(\mathfrak{a}), \mathfrak{g}$ a finite dimensional Lie algebra which contains $\mathfrak{a}$.

Proposition 3.1. Let $A, B \in \mathscr{J}(\mathfrak{a})$ and let $\xi_{n}(n \in N)$ be nonzero constants in $\boldsymbol{R}$. For any $\phi$ in $I_{a}(A, B)$ (resp. $I H_{\mathrm{a}}(A, B)$ ) there exists a unique pairing $C(\varphi)={ }^{\circ}\langle\cdot, \cdot\rangle$ in $I_{a}\left(C(A), C(B)\right.$ ) (resp. $I H_{a}(C(A)$, $C(B))$ ) such that

(i) ${ }^{c}\langle\cdot, \cdot\rangle$ equals zero on $(C(A) \times B) \cup(A \times C(B))$,

(ii) for each $n \in N, a \in C(A)^{X}[n], b \in C(B)^{X}[n]$,

$$
{ }^{c}\langle a, b\rangle=\xi_{n}\left\langle Y^{n+1} a, Y^{n+1} b\right\rangle .
$$

For $\phi$ in $I_{a}(A, A)$, the proposition is precisely Proposition 8.1 [5]. For $\varphi \in I H_{a}(A, A)$, the proof of the proposition is entirely analogous. The case $B \neq A$ is proved exactly as the case $A=B$ and we 
omit the details.

By Proposition 3.1, for each set of nonzero constants $\xi_{n}(n \in N)$ we have the transfer maps for $A, B \in \mathscr{F}(\mathfrak{a})$,

$$
\begin{aligned}
& \varphi \longmapsto C(\varphi) \\
& I_{a}(A, B) \longrightarrow I_{a}(C(A), C(B)) \quad \text { and } \\
& I H_{a}(A, B) \longrightarrow I H_{a}(C(A), C(B)) .
\end{aligned}
$$

Definition 3.2. We say the map $\varphi_{A, B} \mapsto C\left(\varphi_{A, B}\right)$ for $A, B$ in $\mathscr{J}(\mathfrak{a})$ and $\varphi_{A, B} \in I_{a}(A, B)$ (resp. $I H_{a}(A, B)$ ) is compatible with tensoring if for any finite dimensional a-module $F$ and any $\varphi_{F} \in I_{a}(F, F)$ (resp. $\left.I H_{a}(F, F)\right)$

$$
C\left(\varphi_{F} \otimes \varphi_{A, B}\right)=\varphi_{F} \otimes C\left(\varphi_{A, B}\right) .
$$

(Here we identify canonically $C(F \otimes A)$ with $F \otimes C(A)$ and $C(F \otimes B)$ with $F \otimes C(B)$.)

Proposition 3.3. The maps $\varphi \rightarrow C(\varphi)$ given in (3.4) are compatible with tensoring if and only if there is a nonzero constant $\xi$ such that

$$
\xi_{n}=\xi / n !(n+1) ! \quad(n \in N ; 0 !=1) .
$$

In the case $\phi \in I_{a}(A, A)$, the proposition is precisely Proposition 8.4 [5] and for $\varphi \in I H_{a}(A, A)$ the proof of the proposition is entirely analogous. The proof in the case $A \neq B$ is exactly the same as the case $A=B$ and we omit the details.

Now we consider the relative situation $\mathfrak{a} \subseteq \mathrm{g}$. Fix the constants $\xi_{n}=1 / n !(n+1) !(n \in N)$ and let $\varphi \mapsto C(\varphi)$ be the map given by (3.1) for this choice of constants.

TheOREM 3.4. Let $A, B \in \mathscr{F}_{\mathrm{g}}(\mathfrak{a})$ and let $\varphi \in I_{\mathrm{g}}(A, B)$ (resp. $I H_{\mathrm{g}}(A$, $B)$ ). Then $C(\varphi) \in I_{\mathrm{g}}(C(A), C(B))$ (resp. $I H_{\mathrm{g}}(C(A), C(B))$ ).

For $\varphi \in I_{8}(A, A)$ this theorem is precisely Proposition 8.5 [5]. The proof given here is different from the proof in [5], is more conceptual and is based on properties of certain vector valued pairings. Before giving the proof, we establish a few properties of vector valued pairings.

For g-modules $A, B$ and $F$, we call $\psi$ an invariant pairing (resp. invariant Hermitian pairing) of $A$ and $B$ with values in $F$ if $\psi$ is a bilinear (resp. Hermitian) map $\psi: A \times B \rightarrow F$ and, for $x \in U(\mathfrak{g}), a \in A$, $b \in B, \psi(x \cdot a, b)-\psi\left(a, x^{\sigma_{\mathfrak{B}}} \cdot b\right)=x \cdot \psi(a, b)\left(\right.$ resp. $\psi(x \cdot a, b)-\psi\left(a, x^{\bar{\sigma}_{\mathrm{B}}} \cdot b\right)=$ $x \cdot \psi(a, b)$ ). Denote the set of such pairings by $I_{8}(A, B, F)$ (resp. 
$\left.I H_{\mathfrak{g}}(A, B, F)\right)$. If $F$ is the trivial module then $I_{\mathfrak{g}}(A, B, F)=I_{\mathfrak{g}}(A, B)$ and $I H_{8}(A, B, F)=I H_{8}(A, B)$.

Next we shall associate to any pairing $\psi$ two scalar valued pairings. For any vector space $V$, let $V^{*}$ denote the algebraic dual of $V$. Let $\bar{V}$ be the set $V^{*}$ with multiplication by elements of $C$ denoted by $*$ and given by $\alpha * \lambda=\bar{\alpha} \lambda, \alpha \in C, \lambda \in V^{*} . \bar{V}$ is a vector space over $C$ called the conjugate dual of $V$. If $V$ is a g-module, then $\bar{V}$ becomes a $\mathrm{g}$-module by composing the $\boldsymbol{R}$-linear automorphism $-\bar{\sigma}$ and the contragradient representation of $\mathfrak{g}$ on $V^{*}$. We shall call this the conjugate dual module to $V$ and denote it by $\bar{V}$. For $\psi \in I_{8}(A, B, F)$ define $\psi^{R} \in I_{3}\left(A, F^{*} \otimes B\right)$ and ${ }^{L} \psi \in I_{8}\left(F^{*} \otimes A, B\right)$ by the formulae: for $\lambda \in F^{*}, a \in A, b \in B$,

$$
\begin{aligned}
& \psi^{R}(a, \lambda \otimes b)=\lambda(\psi(a, b)), \\
& { }^{L} \psi(\lambda \otimes a, b)=\lambda(\psi(a, b)) .
\end{aligned}
$$

If $\psi \in I H_{8}(A, B, F)$ then define $\psi^{R} \in I H_{8}(A, \bar{F} \otimes B)$ and ${ }^{L} \psi \in I H_{8}\left(F^{*} \otimes\right.$ $A, B)$ by the formulae (3.2) and (3.3) respectively. We note that if $F$ is finite dimensional then $\psi$ is determined by $\psi^{R}$ and by ${ }^{L} \psi$.

Assume $F$ is finite dimensional and $A, B \in \mathscr{F}(\mathfrak{a})$. Using $\psi^{R}$ and ${ }^{L} \psi$ we define two transfers of $\psi$ as follows. For $\psi \in I_{\mathrm{a}}(A, B, F)$ (resp. $I H_{a}(A, B, F)$ ), define $R C(\psi)$ and $L C(\psi)$ to be the unique elements of $I_{a}(C(A), C(B), F) \quad$ (resp. $\quad I H_{a}(C(A), C(B), F)$ ) such that $(R C(\psi))^{R}=C\left(\psi^{R}\right)$ and ${ }^{L}(L C(\psi))=C\left({ }^{L} \psi\right)$.

Proposition 3.5. For $\psi \in I(A, B, F)$ or $I H(A, B, F), R C(\psi)=$ $L C(\psi)$

Proof. First assume $\psi \in I_{8}(A, B, F)$. Let $\phi$ be the canonical pairing of $F$ and $F^{*}$. Tensoring we obtain a pairing

$$
\varphi \otimes{ }^{L} \gamma:\left(F \otimes F^{*} \otimes A\right) \times\left(F^{*} \otimes B\right) \longrightarrow C .
$$

Since $F \otimes F^{*}$ is canonically isomorphic to $\operatorname{Hom}\left(F^{*}, F^{*}\right)$, the identity element of $\operatorname{Hom}\left(F^{*}, F^{*}\right)$ induces a canonical inclusion:

$$
i: A \longrightarrow F \otimes F^{*} \otimes A \text {. }
$$

Via this inclusion, we restrict $\varphi \otimes{ }^{L} \psi$ to obtain the pairing:

$$
\operatorname{Res}\left(\varphi \otimes{ }^{L} \psi\right): A \times\left(F^{*} \otimes B\right) \longrightarrow C .
$$

With an easy calculation, one verifies that " $\psi$ and hence $\psi$ is determined by $\operatorname{Res}\left(\varphi \otimes{ }^{L} \psi\right)$; and moreover, if $\psi_{i} \in I_{\mathrm{g}}(A, B, F), \quad(i=1,2)$, then

$$
\psi_{1}=\psi_{2} \Longleftrightarrow \operatorname{Res}\left(\varphi \otimes{ }^{L} \psi_{1}\right)=\psi_{2}^{R}
$$


Assertion (3.7) shows that to prove $L C(\psi)=R C(\psi)$ we need only prove $\operatorname{Res}\left(\varphi \otimes{ }^{L}(L C(\psi))\right)=(R C(\psi))^{R}$. Thus we need only prove the identity:

$$
\operatorname{Res}\left(\varphi \otimes C\left({ }^{L} \psi\right)\right)=C\left(\psi^{R}\right) .
$$

By Proposition 3.3, $\varphi \otimes C\left({ }^{L} \psi\right)=C\left(\varphi \otimes{ }^{L} \psi\right)$ and by functoriality of $C(\cdot)$ applied to (3.5), $C(i)$ is the canonical inclusion $C(A) \rightarrow F \otimes F^{*} \otimes$ $C(A)$. This implies: $\operatorname{Res}\left(\varphi \otimes C\left({ }^{L} \psi\right)\right)=C\left(\operatorname{Res}\left(\varphi \otimes{ }^{L} \psi\right)\right)$. However, by (3.7), $\operatorname{Res}\left(\varphi \otimes{ }^{L} \psi\right)=\psi^{R}$; and so, the identity (3.8) is established. This completes the proof for $\psi \in I_{8}(A, B, F)$. If $\psi \in I H_{8}(A, B, F)$ then since the map $\varnothing$ gives an invariant Hermitian pairing of $F$ and $\bar{F}$ the same argument applies and the proof is complete.

Proof of Theorem 3.4. For any $\varphi \in I H_{\mathfrak{a}}(A, B)$ with $A, B \in \mathscr{F}_{\mathrm{g}}(\mathfrak{a})$, define $\mathrm{g}^{*}$ valued pairings ${ }_{L} \mathscr{\varphi}$ and $\varphi_{R}$ by the formulae: for $X \in \mathfrak{g}$, $a \in A, b \in B$,

$$
\begin{aligned}
& { }_{L} \varphi(a, b)(X)=\varphi(X \cdot a, b) \\
& \varphi_{R}(a, b)(X)=\varphi\left(a, X^{\sigma_{8}} \cdot b\right) .
\end{aligned}
$$

One checks that ${ }_{L} \varphi$ and $\varphi_{R}$ are elements of $I H_{\mathrm{a}}\left(A, B, \mathrm{~g}^{*}\right)$. We now claim the following identities hold:

$$
\begin{aligned}
& { }^{L}\left({ }_{L} C(\varphi)\right)=C\left({ }^{L}\left({ }_{L} \varphi\right)\right) \\
& \left(C(\varphi)_{R}\right)^{R}=C\left(\left(\varphi_{R}\right)^{R}\right) .
\end{aligned}
$$

Let $\pi$ be the a-module map: $\pi: \mathfrak{g} \otimes A \rightarrow A, X \otimes a \mapsto X \cdot a,(X \in \mathfrak{g}, a \in A)$ and $\pi \times 1:(g \otimes A) \times B \rightarrow A \times B$. Then $\left.{ }^{L}{ }_{L} \varphi\right)=\varphi \circ(\pi \times 1)$; and so, $C\left({ }^{L}\left({ }_{L} \varphi\right)\right)=C(\varphi) \circ(C(\pi) \times 1)$. By uniqueness of $C(\pi), C(\pi)(X \otimes a)=X \cdot a$ $(X \in \mathfrak{g}, a \in C(A))$; and thus, identity (3.10) is true. Identity (3.11) is proved in essentially the same way. Combining (3.3), (3.10) and (3.2), (3.11), we obtain:

$$
\begin{aligned}
& L C\left({ }_{L} \varphi\right)={ }_{L} C(\varphi) \\
& R C\left(\varphi_{R}\right)=C(\varphi)_{R} .
\end{aligned}
$$

Now assume $\phi \in I H_{8}(A, B)$. Invariance is equivalent to saying that ${ }_{L} \varphi$ equals $\varphi_{R}$. By Proposition 3.5 and identities (3.12) and (3.13) we have: ${ }_{L} C(\varphi)=C(\varphi)_{R}$. This says that $C(\varphi)$ is invariant.

In the case where $\phi \in I_{8}(A, B)$ the argument is essentially the same. We need only replace $\bar{\sigma}_{8}$ by $\sigma_{8}$ in definition (3.9). We omit the details.

4. Pairings and lattices. Let notation be as in $\S 1$. In particular, $\mathfrak{m}$ is a reductive Lie algebra over $C$ which is contained in 
a finite dimensional Lie algebra $g$. Let $t_{0} \in \mathscr{W}$ be the unique element such that $t_{0} Q=-Q$.

LEMmA 4.1. Let $t_{0}=s_{\alpha_{1}} \cdots s_{\alpha_{d}}=s_{\beta_{1}} \cdots s_{\beta_{d}}$ be two reduced expressions for $t_{0}\left(\alpha_{i}, \beta_{j}\right.$ simple $)$. For any Q-dominant integral weight $\mu$, set

$$
m_{i}=\left(s_{\alpha_{i-1}} \cdots s_{\alpha_{1}}(\mu+\delta)\right)\left(\bar{H}_{\alpha_{i}}\right), \quad n_{i}=\left(s_{\beta_{i-1}} \cdots s_{\beta_{1}}(\mu+\delta)\right)\left(\bar{H}_{\beta_{i}}\right)
$$

Then, in $U\left(\mathfrak{n}^{-}\right)$we have the identity:

$$
X_{-\alpha_{d}}^{m_{d}} \cdots X_{-\alpha_{1}}^{m_{1}}=X_{-\beta_{d}}^{n_{d}} \cdots X_{-\beta_{1}}^{n_{1}} .
$$

Proof. Let $x$ (resp. $y$ ) denote the left (resp. right) side of (4.1). $x \otimes 1$ and $y \otimes 1$ both span the space of $\mathfrak{n}$-invariants of weight $t_{0}(\mu+\delta)-\delta$ in $V_{\mathrm{m}, Q, \mu}$; and so, $x$ is a nonzero multiple of $y$. However, since the simple roots are linearly independent and $\sum m_{i} \alpha_{i}=$ $\mu-t_{0}(\mu+\delta)+\delta=\sum n_{i} \beta_{i}$, identity (4.1) is true in the symmetric algebra. This implies $x-y$ is an element of $U^{r-1}(\mathfrak{m}), r=\sum m_{i}=$ $\sum n_{i}$. But $x$ is a nonzero multiple of $y$ and $x, y \notin U^{r-1}(\mathfrak{m})$ so $x=y$.

Proposition 4.2. Let $t_{0}=s_{\alpha_{1}} \cdots s_{\alpha_{d}}=s_{\beta_{1}} \cdots s_{\beta_{d}}$ be two reduced expressions for $t_{0}\left(\alpha_{i}, \beta_{i}\right.$ simple). Let $A, B \in \mathscr{F}_{\mathfrak{g}}(\mathfrak{m})$ and $\varphi \in I_{\mathfrak{g}}(A, B)$ or $I_{H_{s}}(A, B)$. Then the pairings $C_{\alpha_{1}} \circ \cdots \circ C_{\alpha_{d}}(\varphi)$ and $C_{\beta_{1}} \circ \cdots \circ C_{\beta_{d}}(\varphi)$ are equal and this pairings is zero on $\left(\sum_{s \neq 1} A_{s} \times B_{1}\right) \cup\left(A_{1} \times \sum_{s \neq 1} B_{s}\right)$.

Proof. Assume $\phi \in I_{\theta}(A, B)$. Let $\varphi_{r_{, i}}=C_{\gamma_{i}} \circ \cdots \circ C_{\gamma_{d}}(\varphi), \quad(\gamma=\alpha$ or $\beta)$. Clearly $\varphi_{r, 1} \in I_{8}\left(A_{1}, B_{1}\right)$ and, directly from the definition, $\varphi_{\alpha, 1}$ is zero on $\left(A_{s_{\alpha_{1}}} \times B\right) \cup\left(A \times B_{s_{\alpha_{1}}}\right)$. For any simple root $\xi$, we have (Theorem 1 [2]):

$$
s_{\xi} t_{0}=s_{\alpha_{1}} \cdots \hat{s}_{\alpha_{j}} \cdots s_{\alpha_{d}} \text { for some } j, \quad 1 \leqq j \leqq d,
$$

(^ denotes omission).

Set $A_{i}=C_{\alpha_{i}} \circ \cdots \circ C_{\alpha_{d}}(A), B_{i}=C_{\alpha_{i}} \circ \cdots \circ C_{\alpha_{d}}(B),(1 \leqq i \leqq d)$. By (4.2), the restriction of $\varphi_{\alpha, 1}$ to $\left(A_{s_{\xi}} \times B_{1}\right) \cup\left(A_{1} \times B_{\xi_{\xi}}\right)$ equals $C_{\alpha_{1}} \circ \cdots \circ C_{\alpha_{j-1}}$ of $\phi_{\alpha, j}$ restricted to $\left(A_{j+1} \times B_{j}\right) \cup\left(A_{j} \times B_{j+1}\right)$. But $\varphi_{\alpha, j}$ is zero on $\left(A_{j+1} \times B_{j}\right) \cup$ $\left(A_{j} \times B_{j+1}\right)$; and so, $\varphi_{\alpha, 1}$ is zero on $\left(\sum_{s \neq 1} A_{s} \times B_{1}\right) \cup\left(A_{1} \times \sum_{s \neq 1} B_{s}\right)$. The same argument applies to $\varphi_{\beta, 1}$. Therefore both $\varphi_{\alpha, 1}$ and $\varphi_{\beta, 1}$ induce invariant pairings on $\tau(A) \times \tau(B)$. These modules are $U(\mathfrak{m})$ finite; and so, we need only check that for $Q$-dominant integral $\mu$ and n-invariant vectors $z \in A_{1}, w \in B_{1}$ of weight $\mu$

$$
\varphi_{\alpha, 1}(z, w)=\varphi_{\beta, 1}(z, w) \text {. }
$$


Using the definitions directly we obtain:

$$
\begin{aligned}
& \varphi_{\alpha, 1}(z, w)=\prod_{i} \frac{1}{m_{i} !\left(m_{i}+1\right) !} \varphi\left(X_{-\alpha_{d}}^{m_{d}} \cdots X_{-\alpha_{1}}^{m_{1}} z, X_{-\alpha_{d}}^{m_{d}} \cdots X_{-\alpha_{1}}^{m_{1}} w\right) \\
& \varphi_{\beta, 1}(z, w)=\prod_{i} \frac{1}{n_{i} !\left(n_{i}+1\right) !} \varphi\left(X_{-\beta_{d}}^{n_{d}} \cdots X_{-\beta_{1}}^{n_{1}} z, X_{-\beta_{d}}^{n_{d}} \cdots X_{-\beta_{1}}^{n_{1}} w\right) .
\end{aligned}
$$

Now, since $\left\{m_{i}: 1 \leqq i \leqq d\right\}=\left\{n_{i}: 1 \leqq i \leqq d\right\}=\left\{\mu+\delta\left(\bar{H}_{\gamma}\right): \gamma \in Q\right\}$, identities (4.1) and (4.4) imply (4.3) and the proof is complete for $\varphi \in I_{8}(A, B)$. The case $\varphi \in I H_{8}(A, B)$ is handled by the same argument.

Definition 4.3. With notation as in Proposition 4.2, the invariant pairing $C_{\alpha_{1}} \circ \cdots \circ C_{\alpha_{d}}(\varphi)$ on $A_{1} \times B_{1}$ is independent of the reduced expression and induces a pairing on $\tau(A) \times \tau(B)$, which we denote by $\tau(\varphi)$. We have:

$$
\begin{aligned}
& \tau: I_{\mathrm{g}}(A, B) \longrightarrow I_{\mathrm{g}}(\tau(A), \tau(B)), \\
& \tau: I H_{\mathrm{g}}(A, B) \longrightarrow I H_{\mathrm{g}}(\tau(A), \tau(B)) .
\end{aligned}
$$

Next we consider an important example where the maps $\tau$ in (4.5) are surjective. Let $d_{t_{0}, 1}(\mu)$ denote the element of $U\left(\mathfrak{n}^{-}\right)$given in the identity (4.1).

LEMmA 4.4. Let $E$ and $F$ be finite dimensional m-modules and let $\nu$ and $\xi$ be $-Q$-dominant integral elements of $\mathfrak{h}^{*}$. Let $A=E \otimes$ $V_{\mathrm{m}, Q, \nu-\delta}$ and $B=F \otimes V_{\mathrm{m}, Q, \hat{\xi}-\dot{\delta}}$. Then the maps $\tau$ in (4.5) are surjective.

Proof. Let $A_{s}(s \in \mathscr{Y})$ and $B_{s}(s \in \mathscr{W})$ be lattices above $A$ and $B$ respectively. Replacing $A$ and $B$ by summands we assume that for some $\chi, \chi^{\prime} \in Z(\mathfrak{m})^{\wedge}, A=A_{\chi}$ and $B=B_{\chi^{\prime}}$. Since generalized $Z(\mathfrak{m})$ eigenspaces for distinct characters are orthogonal it is sufficient to prove the lemma in the setting where $\chi=\chi^{\prime}$. Choose $\mu \in \mathfrak{h}^{*}$ such that $\mu+\delta$ is $Q$-dominant integral and $\chi$ is parameterized by the orbit $\mathscr{W} \cdot(\mu+\delta)$.

By Lemma 7 [4], the maps $z \rightarrow \bar{z}$ of $A_{1}^{\mathfrak{n}}$ to $\tau(A)^{\mathfrak{n}}, B_{1}^{\mathfrak{n}}$ to $\tau(B)^{\mathfrak{n}}$ are surjective. Choose subspaces $M_{1} \subseteq A^{\mathrm{n}}[\mu], N_{1} \subseteq B_{1}^{\mathrm{n}}[\mu]$ such that the induced maps give bijections $M_{1} \underset{\rightarrow}{\sim} \tau(A)^{\mathfrak{n}}, N_{1} \widetilde{\rightarrow} \tau(B)^{\mathrm{n}}$. Define linear subspaces $M=d_{t_{0,1}}(\mu) \cdot M_{1}, N=d_{t_{0,1}}(\mu) \cdot N_{1}$. Then $M$ and $M_{1}$ (resp. $N$ and $N_{1}$ ) are linearly isomorphic and $M \cong A^{\mathrm{n}}\left[t_{0}^{\prime} \mu\right], N \cong B^{\mathrm{n}}\left[t_{0}^{\prime} \mu\right]$. Let $\bar{\varphi} \in I_{\mathrm{g}}(\tau(A), \tau(B))$. Clearly, $\tau(A)$ and $\tau(B)$ being semisimple, $\bar{\varphi}$ is determined by its restriction to $\tau(A)^{\mathfrak{n}} \times \tau(B)^{\mathfrak{n}}$. Let $\psi_{1}$ denote the pull back of this restriction to $M_{1} \times N_{1}$ and define $\psi$ on $M \times N$ by the formula: $\psi\left(d_{t_{0,1}}(\mu) a, d_{t_{0,1}}(\mu) b\right)=\psi_{1}(a, b), a \in M_{1}, b \in N_{1}$. We claim that there exist submodules $A^{\prime} \subseteq A, B^{\prime} \cong B$ such that:

$$
A=A^{\prime} \oplus U(\mathfrak{m}) M, \quad B=B^{\prime} \oplus U(\mathfrak{m}) N .
$$


Assume, for the moment, $A^{\prime}$ and $B^{\prime}$ exist and (4.6) holds. $U(\mathfrak{m}) M$ and $U(\mathfrak{m}) N$ are the direct sums of irreducible Verma modules all of highest weight $t_{0}^{\prime} \cdot \mu$. Now Proposition 6.12 [5] implies that there exists an invariant pairing $\varphi$ of $U(\mathfrak{m}) M$ and $U(\mathfrak{m}) N$ which restricts to $\psi$ on $M \times N$. We extend $\varphi$ to $A \times B$ by setting it equal to zero on $\left(A^{\prime} \times B\right) \cup\left(A \times B^{\prime}\right)$. But then, for some nonzero constant $\Gamma, \bar{\varphi}=$ $\tau(\Gamma \cdot \varphi)$. Thus to complete the proof of surjectivity of $I_{8}(A, B)$ onto $I_{8}(\tau(A), \tau(B))$ we need only prove (4.6) holds for some submodules $A^{\prime}$ and $B^{\prime}$.

Let $\varphi_{E}$ be a nondegenerate invariant form on $E$ and let $\varphi_{\nu}$ be a nondegenerate invariant form on $V_{\mathrm{m}, Q, \nu-\delta}$ (cf. Proposition 6.8 [5]). $\tau\left(\phi_{\nu}\right)$ is a nonzero invariant form on $\tau\left(V_{\mathrm{m}, Q, \nu-\delta}\right)$. But this module is the irreducible m-module with highest weight $t_{0}(\nu)-\delta$; and so, $\tau\left(\varphi_{\nu}\right)$ is nondegenerate. Then $\tau\left(\varphi_{E} \otimes \varphi_{\nu}\right)=\varphi_{E} \otimes \tau\left(\varphi_{\nu}\right)$ is nondegenerate. Now $\tau\left(\varphi_{E} \otimes \varphi_{\nu}\right)$ restricted to $\tau(A)^{\mathfrak{n}} \times \tau(A)^{\mathfrak{n}}$ is nondegenerate; and therefore, $\varphi_{E} \otimes \varphi_{\nu}$ restricted to $M \times M$ is nondegenerate. So since $U(\mathfrak{m}) M$ is the direct sum of irreducible Verma modules $\varphi_{E} \otimes \varphi_{\nu}$ restricted to $U(\mathfrak{m}) M \times U(\mathfrak{m}) M$ is nondegenerate. We put $A^{\prime}$ equal to the orthogonal complement to $U(\mathfrak{m}) M$ in $A$ with respect to $\varphi_{E} \otimes \varphi_{\nu}$. The argument for $B$ is identical; and so, the proof of (4.6) is complete.

For the case of invariant Hermitian pairings we note that if $\lambda \in \mathfrak{h}^{*}$ is real valued on $\bar{H}_{\alpha}(\alpha \in Q)$, and $V_{\mathrm{m}, Q, \lambda}$ is irreducible, then $V_{\mathrm{m}, Q, \lambda}$ and its conjugate dual module (w.r.t. $\bar{\sigma}$ ) are isomorphic. Here we are using the fact that $\bar{\sigma}$ was determined by a compact real form of $\mathfrak{m}$. With this fact in mind, essentially the same argument as above applies to show $\tau: I H_{8}(A, B) \rightarrow I H_{8}(\tau(A), \tau(B))$ is surjective.

For any $\mathfrak{b}$-module $M$, we denote the induced module from $\mathfrak{b}$ to $\mathfrak{m}$ by $U(M)$; i.e., $U(M)=U(\mathfrak{m}) \otimes_{U(\mathfrak{b})} M$.

Proposition 4.5. Let $M$ and $N$ be locally finite b-modules which are semisimple as h-modules and have finite dimensional weight spaces. Assume that $U(M)$ and $U(N)$ admit nondegenerate invariant forms. Then the following maps are surjections:

$$
\begin{aligned}
& \tau: I_{\mathrm{m}}(U(M), U(N)) \longrightarrow I_{\mathrm{m}}(\tau(U(M)), \tau(U(N))) \\
& \tau: I H_{\mathrm{m}}(U(M), U(N)) \longrightarrow I H_{\mathrm{m}}(\tau(U(M), \tau(U(N))) .
\end{aligned}
$$

Moreover, both maps carry nondegenerate pairings to nondegenerate pairings.

Proof. Since $M$ and $N$ are locally finite, $U(M)$ and $U(N)$ are each the direct sums of their generalized $Z(\mathfrak{m})$ eigenspaces $U(M)_{\chi}$ and $U(N)_{x}, \chi \in Z(\mathfrak{m})^{\wedge}$. By assumption the weight spaces of $M$ and $N$ are finite dimensional; and thus, for each $\chi \in Z(\mathfrak{m})^{\wedge}$ there exist finite 
dimensional sub b-modules $M^{\prime} \subseteq M, N^{\prime} \subseteq N$ such that:

$$
U(M)_{\chi} \subseteq U\left(M^{\prime}\right), \quad U(N)_{\chi} \subseteq U\left(N^{\prime}\right) .
$$

For any pairing $\phi \in I_{\mathrm{m}}(U(M), U(N))$ or $I H_{\mathrm{m}}(U(M), U(N))$ and $\chi, \chi^{\prime} \in$ $Z(\mathfrak{m})^{\wedge}, \chi \neq \chi^{\prime}$, we have:

$$
U(M)_{\chi} \subseteq\left(U(N)_{\chi^{\prime}}\right)^{\perp}, \quad U(N)_{\chi^{\prime}} \subseteq\left(U(M)_{\chi}\right)^{\perp} .
$$

The inclusions (4.8) imply that we need only prove the proposition for $U(M)$ replaced by $U(M)_{x}$ and $U(N)$ replaced by $U(N)_{x}$. For convenience we set $A=U(M)_{\chi}$ and $B=U(N)_{\chi}$.

Using Lemma 4.7 [5], choose a finite dimensional m-module $F$ and integral weights $\mu, \nu \in \mathfrak{h}^{*}$ with $\mu\left(\bar{H}_{\alpha}\right) \ll 0, \nu\left(\bar{H}_{\alpha}\right) \ll 0(\alpha \in Q)$, such that we have embeddings:

$$
M^{\prime} \hookrightarrow F \otimes C_{\mu}, \quad N^{\prime} \hookrightarrow F \otimes C_{\nu} .
$$

Extending scalars to $U(\mathfrak{m})$ and setting $V_{\mu}=V_{\mathfrak{m}, Q, \mu}, V_{\nu}=V_{\mathfrak{m}, Q, \nu}$, we obtain embeddings:

$$
U\left(M^{\prime}\right) \smile F \otimes V_{\mu}, \quad U\left(N^{\prime}\right) \hookrightarrow F \otimes V_{\nu} .
$$

By assumption $U(M)$ and hence $A$ admits a nondegenerate invariant form, say $\zeta$. Then using Propositions 6.13 and 6.7 [5] there exists an invariant form $\bar{\zeta}$ on $F \otimes V_{\mu}$ which is nondegenerate and restricts to $\zeta$ on $A$. This implies that $A$ is a direct summand of $F \otimes V_{\mu}$; and so, $\tau(A)$ is a direct summand of $\tau\left(F \otimes V_{\mu}\right)$. The same argument implies that $\tau(B)$ is a summand of $\tau\left(F \otimes V_{\nu}\right)$. But then if Res denotes the restriction map for pairings, the map

$$
\text { Res: } I_{\mathrm{m}}\left(\tau\left(F \otimes V_{\mu}\right), \tau\left(F \otimes V_{\nu}\right)\right) \longrightarrow I_{\mathrm{m}}(\tau(A), \tau(B))
$$

is a surjection. Let $\bar{\phi} \in I_{\mathrm{m}}(\tau(A), \tau(B))$. Then by (4.11), choose an invariant pairing $\bar{\psi}$ such that $\operatorname{Res}(\bar{\psi})=\bar{\varphi}$. By Lemma 4.4, choose $\psi \in I\left(F \otimes V_{\mu}, F \otimes V_{\nu}\right)$ such that $\tau(\psi)=\bar{\psi}$. If $\phi$. denotes the restriction of $\psi$ to $A \times B$, then $\tau(\varphi)=\bar{\varphi}$. This proves surjectivity.

Let $\varphi \in I_{\mathrm{m}}(A, B)$ and assume $\varphi$ is nondegenerate. Using Propositions 6.13 and 6.7 [5], there exists a nondegenerate invariant pairing $\psi$ of $\left(F \otimes V_{\mu}\right) \times\left(F \otimes V_{\nu}\right)$ which restricts to $\varphi$. We use $\psi$ to obtain the orthogonal decomposition:

$$
F \otimes V_{\mu}=A \oplus B^{\perp}, \quad F \otimes V_{\nu}=B \oplus A^{\perp} .
$$

Let $\xi \in \mathfrak{h}^{*}$ be $Q$-dominant integral and let $z_{1}$ be an $\mathfrak{n}$-invariant of weight $\xi$ in $A_{1}$. Assume $z_{1} \notin \sum_{s \neq 1} A_{s}$. From (4.12) we consider $\left(F \otimes V_{\mu}\right)_{s}=A_{s} \oplus B^{\perp}{ }_{s}(s \in \mathscr{W})$; and so, $z_{1} \notin \sum_{s \neq 1}\left(F \otimes V_{\mu}\right)_{s}$. Then, by Proposition 9.8 [5], $z=d_{t_{0,1}}(\xi) \cdot z_{1}$ is a split invariant of $F \otimes V_{\mu}$. By Lemma 9.3 [5] and (4.12), there exists an n-invariant $w$ of weight 
$t_{0}^{\prime} \cdot \xi$ in $B$ such that $\varphi(z, w)=\psi(z, w) \neq 0$. Let $w_{1}$ be the unique $\mathfrak{n}$ invariant in $B_{1}$ such that $w=d_{t_{0,1}}(\xi) \cdot w_{1}$ and let $\bar{z}$ and $\bar{w}$ denote the images of $z_{1}$ and $w_{1}$ in $\tau(A)$ and $\tau(B)$. Then $\tau(\varphi)(\bar{z}, \bar{w}) \neq 0$. This implies that $\bar{z}$ is not contained in $\tau(B)^{\perp}$. But $\bar{z}$ denotes any $\mathfrak{n}$-invariant of $\tau(A)$; and so, $\tau(A) \cap \tau(B)^{\perp}=0$. Likewise $\tau(B) \cap \tau(A)^{\perp}=0$. So $\tau(\varphi)$ is nondegenerate on $A \times B$ and hence on $U(M) \times U(N)$. The argument for $\varphi \in I H_{\mathrm{m}}(U(M), U(N))$ is identical and we omit it.

\section{REFERENCES}

1. A. Bouaziz, Sur les representations des algebra de Lie semi-simples construites par T. Enright, manuscript.

2. V. V. Deodhar, Some characterizations of Bruhat ordering on a Coxeter group and determination of the relative Mobius function, Inventiones Math., 39 (1977), 187-198.

3. - On a construction of representations and a problem of Enright, Invent. Math., 57 (1980), 101-118.

4. T. J. Enright and V.S. Varadarajan, On an infinitesimal characterization of the discrete scries, Ann. of Math., 102 (1975), 1-15.

5. T.J. Enright, On the fundamental series of a real semisimple Lie algebra: their irreducibility, resolutions and multiplicity formulae, Ann. of Math., 109 (1979), $1-82$.

6. - The representations of complex semisimple Lie groups, Tata Institute Lecture Notes.

Received August 9, 1979. Research of the first author was partially supported by N.S.F. grant no. MCS 78-02898.

University of California, San Diego

LA Jolla, CA 92093

AND

Tata Institute of Fundamental Research

Bombay 400 005, India 



\section{PACIFIC JOURNAL OF MATHEMATICS}

\section{EDITORS}

DONALD BABBITT (Managing Editor)

University of California

Los Angeles, CA 90024

HUGo RossI

University of Utah

Salt Lake City, UT 84112

C. C. MOORE and ANDREW OGG

University of California

Berkeley, CA 94720
J. DugundjI

Department of Mathematics

University of Southern California

Los Angeles, CA 90007

R. FinN and J. Milgram

Stanford University

Stanford, CA 94305

\section{ASSOCIATE EDITORS}
R. ARENS
E. F. BECKENBACH
B. H. NeumanN
F. WOLF
K. YOSHIDA

\section{SUPPORTING INSTITUTIONS}

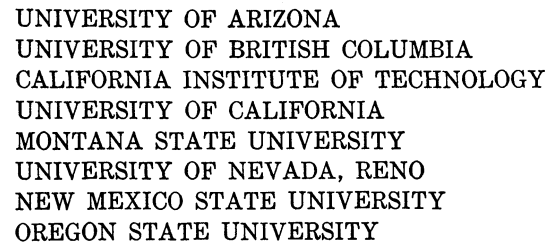

UNIVERSITY OF ARIZONA

UNIVERSITY OF BRITISH COLUMBIA

CALIFORNIA INSTITUTE OF TECHNOLOGY

UNIVERSITY OF CALIFORNIA

MONTANA STATE UNIVERSITY

UNIVERSITY OF NEVADA, RENO

NEW MEXICO STATE UNIVERSITY

OREGON STATE UNIVERSITY

\author{
UNIVERSITY OF OREGON \\ UNIVERSITY OF SOUTHERN CALIFORNIA \\ STANFORD UNIVERSITY \\ UNIVERSITY OF HAWAII \\ UNIVERSITY OF TOKYO \\ UNIVERSITY OF UTAH \\ WASHINGTON STATE UNIVERSITY \\ UNIVERSITY OF WASHINGTON
}

The Supporting Institutions listed above contribute to the cost of publication of this Journal, but they are not owners or publishers and have no responsibility for its content or policies.

Mathematical papers intended for publication in the Pacific Journal of Mathematics should be in typed form or offset-reproduced, (not dittoed), double spaced with large margins. Please do not use built up fractions in the text of the manuscript. However, you may use them in the displayed equations. Underline Greek letters in red, German in green, and script in blue. The first paragraph or two must be capable of being used separately as a synopsis of the entire paper. Please propose a heading for the odd numbered pages of less than 35 characters. Manuscripts, in triplicate, may be sent to any one of the editors. Please classify according to the scheme of Math. Reviews, Index to Vol. 39. Supply name and address of author to whom proofs should be sent. All other communications should be addressed to the managing editor, or Elaine Barth, University of California, Los Angeles, California, 90024.

50 reprints to each author are provided free for each article, only if page charges have been substantially paid. Additional copies may be obtained at cost in multiples of 50 .

The Pacific Journal of Mathematics is issued monthly as of January 1966. Regular subscription rate: $\$ 102.00$ a year (6 Vols., 12 issues). Special rate: $\$ 51.00$ a year to individual members of supporting institutions.

Subscriptions, orders for numbers issued in the last three calendar years, and changes of address shoud be sent to Pacific Journal of Mathematics, P.O. Box 969, Carmel Valley, CA 93924, U.S.A. Old back numbers obtainable from Kraus Per!odicals Co., Route 100, Millwood, NY 10546.

PUBLISHED BY PACIFIC JOURNAL OF MATHEMATICS, A NON-PROFIT CORPORATION

Printed at Kokusai Bunken Insatsusha (International Academic Printing Co., Ltd.). 8-8, 3-chome, Takadanobaba, Shinjuku-ku, Tokyo 160, Japan. 


\section{Pacific Journal of Mathematics}

\section{Vol. 95, No. $2 \quad$ October, 1981}

George E. Andrews, The Rogers-Ramanujan reciprocal and Minc's

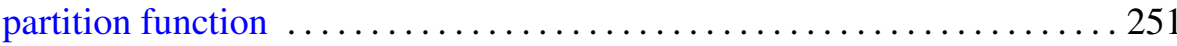

Allan Calder, William H. Julian, Ray Mines, III and Fred Richman,

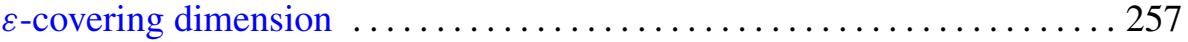

Thomas Curtis Craven and George Leslie Csordas, An inequality for the distribution of zeros of polynomials and entire functions $\ldots \ldots \ldots \ldots 263$

Thomas Jones Enright and R. Parthasarathy, The transfer of invariant

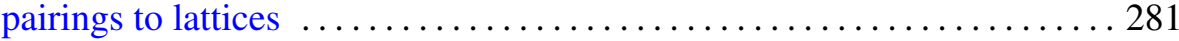

Allen Roy Freedman and John Joseph Sember, Densities and

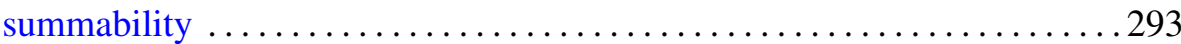

Robert Heller and Francis Aubra Roach, A generalization of a classical necessary condition for convergence of continued fractions . . . . . . 307

Peter Wilcox Jones, Ratios of interpolating Blaschke products ........... 311

V. J. Joseph, Smooth actions of the circle group on exotic spheres ........ 323

Mohd Saeed Khan, Common fixed point theorems for multivalued

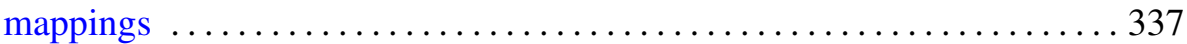

Samuel James Lomonaco, Jr., The homotopy groups of knots. I. How to

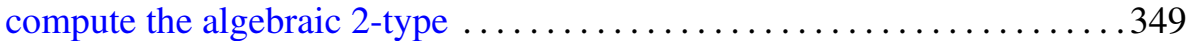

Louis Magnin, Some remarks about $C^{\infty}$ vectors in representations of connected locally compact groups ............................ 391

Mark Mandelker, Located sets on the line . . . . . . . . . . . . . . . . . 401

Murray Angus Marshall and Joseph Lewis Yucas, Linked quaternionic mappings and their associated Witt rings $\ldots \ldots \ldots \ldots \ldots \ldots \ldots \ldots . \ldots \ldots 11$

William Lindall Paschke, $K$-theory for commutants in the Calkin algebra

W. J. Phillips, On the relation $P Q-Q P=-i I$ 435

Ellen Elizabeth Reed, A class of Wallman-type extension. 443

Sungwoo Suh, The space of real parts of algebras of Fourier transforms 461 Antonius Johannes Van Haagen, Finite signed measures on function

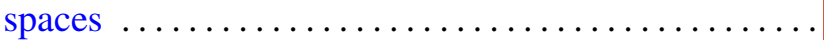

Richard Hawks Warren, Identification spaces and unique uniformity 